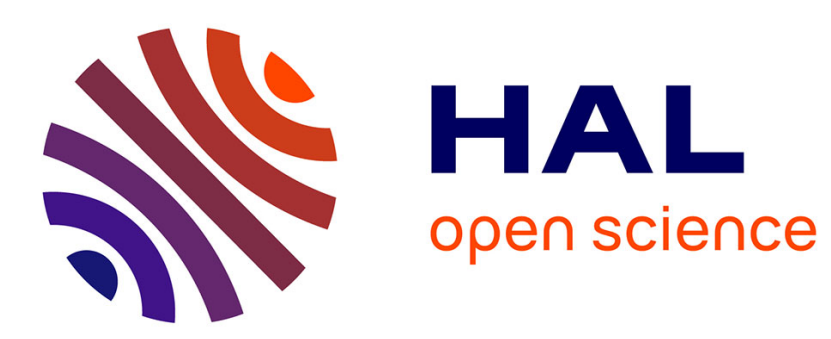

\title{
Liposome Permeability to Essential Oil Components: A Focus on Cholesterol Content
}

Ghenwa Nasr, Hélène Greige-Gerges, Abdelhamid Elaissari, Nathalie Khreich

\section{To cite this version:}

Ghenwa Nasr, Hélène Greige-Gerges, Abdelhamid Elaissari, Nathalie Khreich. Liposome Permeability to Essential Oil Components: A Focus on Cholesterol Content. Journal of Membrane Biology, 2021, 254, pp.381-395. 10.1007/s00232-021-00180-3 . hal-03224970

\section{HAL Id: hal-03224970 \\ https://hal.science/hal-03224970}

Submitted on 3 Nov 2021

HAL is a multi-disciplinary open access archive for the deposit and dissemination of scientific research documents, whether they are published or not. The documents may come from teaching and research institutions in France or abroad, or from public or private research centers.
L'archive ouverte pluridisciplinaire HAL, est destinée au dépôt et à la diffusion de documents scientifiques de niveau recherche, publiés ou non, émanant des établissements d'enseignement et de recherche français ou étrangers, des laboratoires publics ou privés. 


\title{
Liposomal Permeability to Essential oil components : A Focus on Cholesterol Content
}

\author{
Ghenwa Nasr ${ }^{1,2}$, Hélène Greige-Gerges ${ }^{1}$, Abdelhamid Elaissari², Nathalie Khreich ${ }^{\text {* }}$ \\ ${ }^{1}$ Bioactive Molecules Research Laboratory, Faculty of Sciences, Lebanese University, Lebanon. \\ ${ }^{2}$ Laboratoire d'Automatique et de Génie des Procédés (LAGEP), Université Claude Bernard, Lyon \\ 1, France.
}

\section{Abstract}

Encapsulation in liposomes has always been an efficient approach/strategy to improve the stability of sensitive bioactive compounds such as essential oils (EOs) and to increase their biological activities. However, the stability of liposomal formulation remains a major concern for the control of the delivery of encapsulated ingredients. Given the stability that cholesterol (Chol) confers to the membrane, the Chol content used for the preparation of liposomes encapsulating the hydrophobic EO components has to be carefully chosen. In this work, we investigated the effect of Chol content on the permeability of liposome membranes induced by various EO components. Blank liposomes made of DPPC and Chol at different DPPC/Chol molar ratios (100:10; 100:25; $100: 50 ; 100: 75 ; 100: 100)$ were prepared and the final composition of the membrane was determined after extrusion. The same formulations encapsulating the hydrophilic fluorescent agent, sulforhodamine B (SRB), were also prepared and exposed to the EO components at a molar ratio of DPPC:EO of 100:25. The membrane permeability was monitored by following the release of SRB from liposomes with time at $37{ }^{\circ} \mathrm{C}$. Results showed that the DPPC:Chol molar ratios experimentally determined for the formulations exceeding $10 \%$ Chol were lower than the theoretical values. Among the 22 EO components tested, 13 molecules displayed a significant permeabilizing effect on $10 \%$ Chol liposome membranes. Most of these possess a hydroxyl group. The EO induced permeability was also dependent on the Chol content which in turn affects the membrane phase: the EO components effect was reduced upon increasing Chol content from 10 to $21 \%$ Chol ( $\mathrm{S}_{0}-\mathrm{L}_{0}$ phase) keeping 9 molecules effective on the $21 \%$ Chol membranes. At high Chol content where the membrane is in the $\mathrm{L}_{0}$ phase, only five EOs components exerted a permeabilizing effect (menthol, eugenol, thymol, guaiacol and linalool) on 40\% Chol membranes with no effect observed at $43 \%$ and $55 \%$ Chol. The EO's effect was also linked to the hydrophobicity of the molecule: hydrophobic compounds having no hydroxyl group showed a weak permeabilizing effect only in the So-Lo phase. Conversely, when the hydrophobicity of EO component having a hydroxyl group increases, their effect persisted up to higher Chol content. Consequently, the DPPC:Chol ratio of the formulation is chosen taking into account the structure of the compound, its hydrophobicity and its effect on the permeability at different Chol content: a formulation comprising $40 \%$ Chol is suggested for highly hydrophobic molecules whereas a formulation with higher Chol content (more than 40\%) could be selected for less hydrophobic compounds.

Keywords: liposomes, membrane permeability, essential oil components, cholesterol content, hydrophobicity, encapsulation

\section{Highlights}


* EO components bearing a hydroxyl group demonstrated a significant permeabilizing effect on 10\% Chol liposome membranes.

* The permeabilizing effect of EO components was reduced upon increasing Chol content in the $\mathrm{S}_{0}-\mathrm{L}_{0}$ membranes (comprising $10 \%$ and $21 \% \mathrm{Chol}$ )

* A further decrease of the EO components effect was observed in the $\mathrm{L}_{0}$ membranes (composed of $40 \%, 43 \%$ and $55 \%$ Chol) keeping only 5 compounds effective on $40 \%$ Chol membranes

* The increase in the hydrophobicity of the compound having a hydroxyl group reduced its ability to permeabilize the membrane at high Chol content

* The choice of DPPC:Chol ratio for the EO encapsulation depends on the structure of the molecule, its hydrophobicity and its effect on the membrane at various Chol content

\section{Introduction}

Aromatic and medicinal plants are rich in essential oils (EOs), a mixture of natural, volatile, fragrant compounds of low molecular weight, constituting the secondary metabolites of plants. These complex mixtures may comprise 20 to 100 different constituents belonging to a variety of chemical classes generally dominated by: terpenes, phenylpropenes and their oxygenated derivatives as well as other aromatic and aliphatic molecules (Asbahani et al., 2015; Hyldgaard et al., 2012). EOs are used in various sectors, mainly perfumery, cosmetics and food industry (Bakkali et al., 2008; Burt, 2004). They cover a broad biological activity spectrum (Raut and Karuppayil, 2014) including antibacterial (Nazzaro et al., 2013; Zhang et al., 2016), antifungal (Chen et al., 2013; Tian et al., 2012), antioxidant (Miguel, 2010; Teixeira et al., 2013), antiinflammatory (Hajhashemi et al., 2003; Miguel, 2010), antiviral (Astani et al., 2011; Schnitzler et al., 2010) and insecticidal activities (Isman et al., 2008). Besides, some EOs (and some of their constituents) have displayed anticancer (Jaganathan et al., 2011; Kaefer and Milner, 2008) and antidiabetic properties (Boukhris et al., 2012; Chung et al., 2010). Taken all together, EOs are currently used as an alternative natural cure.

Nevertheless, EOs are unstable compounds known for their poor water solubility and their high sensitivity to light, oxygen and temperature which render them prone to degradation (Majeed et al., 2015). Therefore, encapsulation would be a feasible solution to increase EOs stability, enhance their bioavailability, control their release and, hence, improve their efficiency (Asbahani et al., 2015; Sherry et al., 2013). Due to their biocompatibility, biodegradability, low toxicity as well as their ability to entrap hydrophilic, hydrophobic and amphiphilic compounds, liposomes have been 
extensively used as drug carriers (Abu Lila and Ishida, 2017; Sen et al., 2014). During the past 2 decades, efforts have been made to determine the best formulation for the encapsulation of EO components in liposomes. In fact, the lipid composition of the liposomal membrane, dictating the physicochemical properties of the lipid bilayer, affects considerably the stability of the vesicles. In addition, the liposomal formulation controls the release of encapsulated ingredients over time (Bozzuto and Molinari, 2015). The available literature data shows that different liposomal formulations were demonstrated to improve the physicochemical stability of EO constituents and enhance their biological activities; the aforementioned are usually composed of either saturated (dipalmitoylphosphatidylcholine (DPPC), Phospholipon 80H, Phospholipon 90H) or unsaturated phospholipids (phosphatidylcholine (PC), Lipoid S100, Lipoid E80) in combination with cholesterol (Chol) (Azzi et al., 2018; Cui et al., 2017; Gortzi et al., 2007; Lin et al., 2016; Liolios et al., 2009; Moghimipour et al., 2012; Sebaaly et al., 2015b, 2015a). Furthermore, Chol free formulations were also evaluated for their efficiency in encapsulating EOs: it was shown that the choice of the phospholipid to EOs ratio should be taken into account since a large amount of lipophilic components would disturb the lipid bilayer, leading to membrane destabilization (Dag and Oztop, 2017; Detoni et al., 2009; Khosravi-Darani et al., 2016; Yoshida et al., 2010). Consequently, the liposome formulation should be tailored to meet the stability and release requirements of a suitable delivery system.

Liposomal membrane permeability is greatly influenced by the membrane lipid composition. Recently, we have investigated the effect of Chol on the membrane permeability of liposomes composed of various DPPC:Chol molar ratios and encapsulating a hydrophilic fluorescent dye, sulforhodamine B (SRB). The SRB release constants decreased upon increasing Chol content in the liposomal membranes proving thereby the ability of cholesterol to affect the membrane permeability in a dose dependent manner (Kaddah et al., 2018).

Compelling evidence supports the ability of EO components to increase the fluidity and permeability of liposomal membranes by interacting with the bilayer's phospholipids (Cristani et al., 2007; Gharib et al., 2018, 2017; Trombetta et al., 2005). Indeed, some EO components are classified as permeabilizing agents inducing membrane permeabilization via defect formation (Nasr et al., 2020). This may lead to a low encapsulation efficiency due to the escape of the molecules out of the vesicles. To overcome this hurdle, a suitable liposomal formulation has to be 
found where the choice of the phospholipid to Chol ratio is actually a matter of importance. It is worth noting that much of the vast literature on the membrane permeabilizing effect of EO components deals with the antimicrobial effect of EOs where the bacterial cell membranes which are Chol free are used. Hence, in this work, we tested the effect of EO components on the permeability of liposomal membranes made of different DPPC:Chol. A series of 22 EO components was chosen for this study. The membrane permeability will be followed upon exposure of different liposome formulations to these components. This work will allow us to evaluate the effect of Chol on the membrane permeability that could be induced by these components and therefore deduce adequate formulations for EOs encapsulation. A structure activity relationship could be also extracted from the permeability study results.

Blank LUV liposomes were first prepared from a mixture of DPPC and Chol, at different DPPC:Chol molar ratios $(100: 10 ; 100: 25 ; 100: 50 ; 100: 75 ; 100: 100)$ by the reverse phase evaporation method followed by extrusion. Liposomes were then characterized in terms of their size and their lipid composition (DPPC and Chol loading rates and incorporation rates) allowing the determination of the final liposome composition. Similarly, another set of LUVs loaded with SRB was prepared at the same DPPC:Chol molar ratios cited above. These batches were exposed to EO components at a molar ratio DPPC:EO of 100:25 and the SRB release from liposomes was followed during $72 \mathrm{~h}$ at $37^{\circ} \mathrm{C}$ by fluorescence measurements.

Figure 1 shows the chemical structure of the tested molecules. The EO components series is divided into phenolic and non-phenolic compounds: the first group comprises phenylpropanoids, monoterpenes, and phenol ethers. The second group includes monoterpenes bearing different functional groups such as alcohol, ketone and ester. Sesquiterpenes and hydrocarbon monoterpenes are also present in this group. Table 1 in supplementary materials presents the aqueous solubility, the $\log \mathrm{P}$ values and the Henry constants of the tested molecules. 


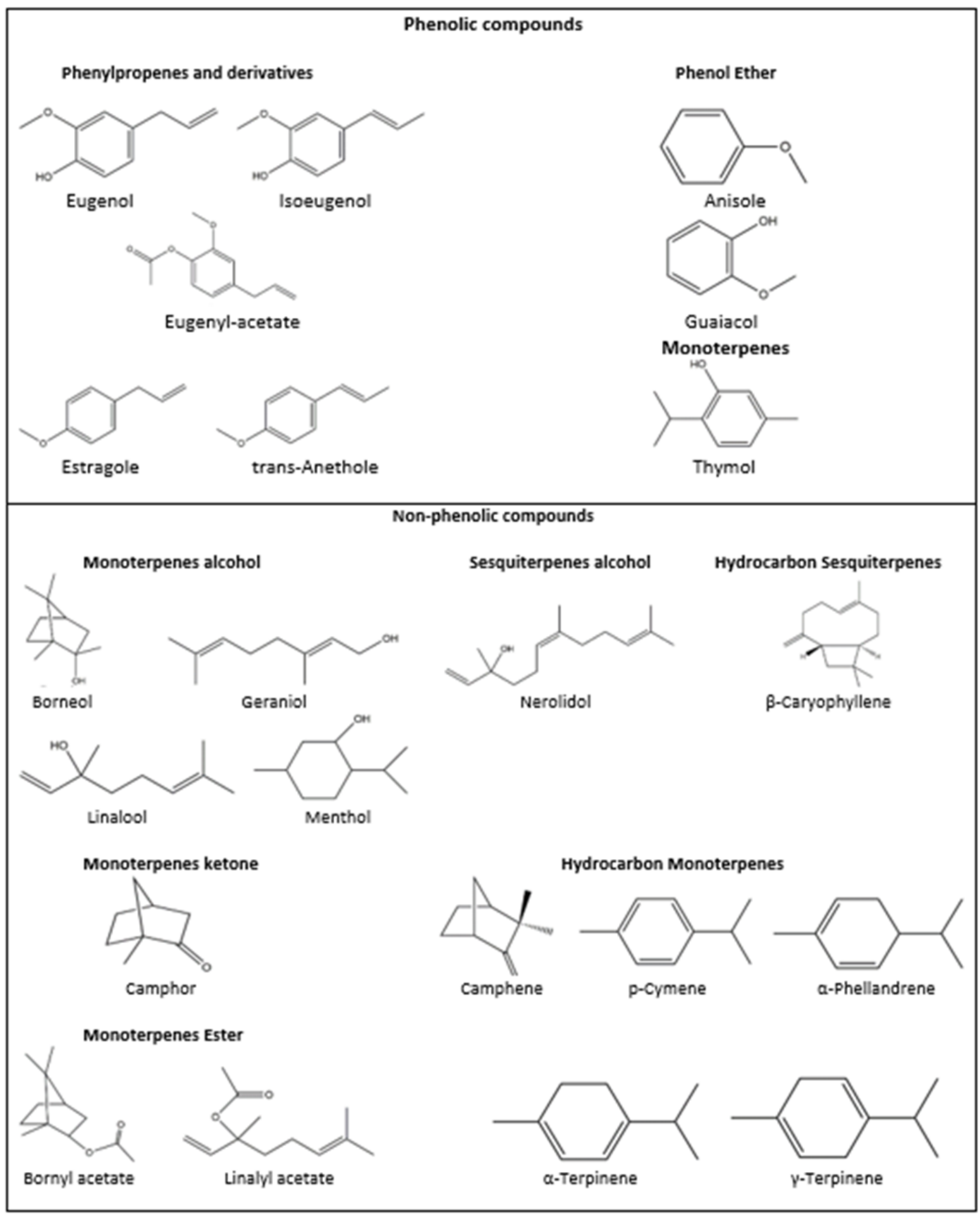

Figure 1: The chemical structures of the 22 EO components tested in this study. 


\section{Materials and Methods}

\subsection{Chemicals}

Dipalmitoylphosphatidylcholine (DPPC) $(\geq 99 \%)$ and trizma base (buffer reagent) were purchased from Sigma-Aldrich, Swiss. Triton X-100, sodium chloride ( $\mathrm{NaCl})$ and Sephadex G25 gel were purchased from Sigma-Aldrich, Belgium. Sodium azide, ammonium molybdate, hydrogen peroxide, potassium dihydrogenophosphate, sodium sulfite, sodium bisulfite, menthol, eugenol, chloroform and methanol were purchased from Sigma-Aldrich, Germany. Linalool was purchased from Sigma-Aldrich, Switzerland. Trans-Anethole was purchased from Sigma-Aldrich, Spain. Geraniol and nerolidol were purchased from Sigma-Aldrich, USA. Sulforhodamin B and camphor were purchased from Sigma-Aldrich, China. Borneol, bornyl acetate, p-cymene, aterpinene and $\mathrm{\gamma}$-terpinene were purchased from Fluka Chemical, Switzerland. 4-amino-3hydroxyl-1-naphtalene sulfonic acid was purchased from Fluka, India. Anisole was purchased from Fluka, Germany. Guaiacol, thymol, estragole, isoeugenol were purchased from ACROS Organics, New Jersey, USA. The sulfuric acid was purchased from ACROS Organics, Belgium. The diethylether was purchased from VWR-Prolabo Chemicals, Belgium. $\beta$-caryophyllene and eugenyl acetate were purchased from SAFC, USA. Linalyl acetate was purchased from SAFC, Spain. a-Phellandrene was purchased from SAFC, Germany. Camphene was purchased from MERCK, Schurchardt.

\subsection{Preparation of large unilamellar vesicles (LUVs) with and without SRB followed by extrusion}

Blank LUVs were prepared by dissolving $25 \mu$ mole of DPPC in $5 \mathrm{ml}$ of a mixture of organic solvents: chloroform/diethyl ether/methanol (6/6/1 - v / v / v) (Khreich et al., 2008). The cholesterol was added at different DPPC: Chol molar ratios (100: 10, 100: 25, 100: 50, 100: 75, 100: 100) from a stock solution of Chol prepared in the same organic solvents mixture $(25 \mathrm{mg} / \mathrm{mL})$. The lipid solution was then sonicated (Sonicator Starsonic 35) for $1 \mathrm{~min}$ at $60^{\circ} \mathrm{C}$ under nitrogen stream. $0.75 \mathrm{ml}$ of Tris $\mathrm{HCl}$ buffer $(0.1 \mathrm{M}, \mathrm{pH}=7.4)$ was added to the mixture, followed by a sonication for 6 minutes at $60^{\circ} \mathrm{C}$ under nitrogen stream. The organic solvents were then removed by evaporation at $45^{\circ} \mathrm{C}$ using a rotary vacuum evaporator (Heidolph, Germany). A white lipid film 
was obtained. In the last step, $1.5 \mathrm{ml}$ of Tris HCI buffer $(0.1 \mathrm{M}, \mathrm{pH}=7.4)$ was added and the mixture was sonicated again for 5 minutes at $60^{\circ} \mathrm{C}$ under nitrogen stream. The obtained liposomal suspensions were stored in the refrigerator at $4{ }^{\circ} \mathrm{C}$ for further manipulation.

Before extrusion, the liposomes were incubated in a water bath for $35 \mathrm{~min}$ at $60^{\circ} \mathrm{C}$ and then extruded through polycarbonate filters, using a mini extruder (Avanti Polar Lipids, Switzerland). Liposomes were forced to pass through polycarbonate membranes (Avanti Polar Lipids, Switzerland) of decreasing pore size ( 5 times through a $1 \mu \mathrm{m}$ membrane followed by 5 times through $0,4 \mu \mathrm{m}$ membrane). This step is important because it allows obtaining a homogenous mixture of LUV liposomes with a mean diameter theoretically very close to the pore size of the last membrane they were extruded through.

The same procedure was followed to prepare and extrude the SRB-loaded LUVs composed of DPPC:Chol molar ratios $(100: 10 ; 100: 25 ; 100: 50 ; 100: 75$ and 100:100). The only difference is that the SRB was added to the aqueous phase composed of Tris $\mathrm{HCl}(0.1 \mathrm{M}, \mathrm{pH}=7.4)$ buffer at a concentration of $150 \mathrm{mM}$. After extrusion, Tris-HCI $(0.1 \mathrm{M}, \mathrm{pH}=7.4)$ containing $150 \mathrm{mM} \mathrm{NaCl}$ was used to dilute the liposomes encapsulating SRB since it maintains the osmolarity of the medium and the integrity of the liposomes, preventing their burst and breakage.

\subsection{Characterization of liposomes}

\subsubsection{Size analysis of blank liposomes}

The mean particle size of blank liposomes was determined by laser granulometry using a Partica LA- 950V2 Lazer Diffraction Particle Size Distribution Analyser (Horiba, Japan) that can detect sizes between $0.01 \mu \mathrm{m}$ and $3000 \mu \mathrm{m} .2 \mathrm{~mL}$ of each liposomal suspension obtained after extrusion was used for the size measurement.

\subsubsection{Determination of liposome composition in the final liposomal structure}

\subsubsection{Separation of loaded and unloaded lipids by centrifugation}

After a dilution of 5 times of the initial liposomal batches, aliquots of the blank LUVs of each formulation underwent an ultrafiltration on Vivaspin 500 tubes during $1 \mathrm{~h}$. This step allows the 
separation of lipids (phospholipids and cholesterol molecules) incorporating the liposome structure from free lipid molecules present in the filtrate.

\subsubsection{Determination of lipids incorporation rate and loading rate in blank liposomes}

\section{Phospholipids (DPPC)}

According to the protocol previously described by (Habib et al., 2013), the total and free amounts of phospholipids (DPPC) were determined for each liposomal suspension using Bartlett method (Bartlett G, 1959). The total DPPC concentration was quantified for the various formulations after adequate dilutions of the initial liposome suspensions whereas the concentration of free DPPC was determined in the filtrates. The DPPC loading rate (LR) was then calculated by the following equation:

$L R=\frac{(m \text { total } D P P C-m \text { free } D P P C)}{m \text { theoretical weighed } D P P C} \times 100$

Where: "m total DPPC" is the calculated mass of total DPPC found in the liposomal suspension, "m free DPPC" is the calculated mass of free DPPC that are not incorporated in the liposomal formulation and are present in the filtrates and " $m$ theoretical DPPC" is the weighed mass of DPPC used for the preparation of liposomes.

The DPPC incorporation rate (IR) was also calculated as follows:

$I R=\frac{m \text { total } D P P C-m \text { free } D P P C}{m \text { total } D P P C} \times 100$

Where: "m total DPPC" is the calculated mass of total DPPC found in the liposomal suspension and "m free DPPC" is the calculated mass of free DPPC that are not incorporated in the liposomal formulation and are present in the filtrates.

\section{Cholesterol}

Chol dosage was performed after an enzymatic hydrolysis and an oxidation using a Chol kit (Cholesterol, spinreact, S.A.U., Spain). $1000 \mu \mathrm{l}$ of the standard kit are added to the $10 \mu 1$ of a solution containing Chol, and incubated for $10 \mathrm{~min}$ at room temperature. Then, the absorbance is 
read at a wavelength of $505 \mathrm{~nm}$. Based on a calibrator concentration of $2 \mathrm{mg} / \mathrm{ml}$, the concentration of Chol $(\mathrm{mg} / \mathrm{dL})$ is given by the following equation:

$$
[\text { Chol }]=\frac{\text { A sample }- \text { A blank }}{\text { A calibrator }- \text { A blank }} \times 200
$$

To determine the total Chol concentration in each batch, $10 \mu 1$ of the liposomal suspension (after adequate dilutions) is sonicated for 20 minutes to ensure the degradation of the liposomes and the release of the total amount of cholesterol encapsulated in the membrane. As for free Chol, $10 \mu 1$ of the filtrates, obtained after centrifugation on vivaspin 500 tubes, is used for the dosage. The absorbance is measured using a spectrophotometer. Measurements were performed in triplicate. The Chol loading rate was calculated according to the following formula:

$L R=\frac{(m \text { total Chol }-m \text { free Chol })}{m \text { theoretical Chol }} \times 100$

Where: "m total Chol" is the calculated mass of total Chol found in the liposomal suspension, "m free Chol" is the calculated mass of free Chol that are not incorporated in the liposomal formulation and are present in the filtrates and " $\mathrm{m}$ theoretical Chol" is the mass of Chol used for the preparation of liposomes.

The Chol incorporation rate for each liposomal formulation was calculated as follows:

$I R=\frac{m \text { total Chol }-m \text { free Chol }}{m \text { total Chol }} \times 100$

Where: "m total Chol" is the calculated mass of total Chol found in the liposomal suspension, "m free Chol" is the mass of free Chol that are not incorporated in the liposomal formulation and are present in the filtrates.

The results are expressed as the means of three independent experiments $\pm \mathrm{SD}$.

\subsubsection{Determination of the DPPC to Chol molar ratio for each formulation}

The DPPC and Chol incorporation rates calculated for blank liposomal batches are used to determine the number of moles of DPPC and Chol incorporated into the liposomes of each formulation.

Then, the DPPC:Chol molar ratios were obtained after determining the percentage of Chol incorporated in the liposome formulation according to the following equation: 
Incorporated Cholesterol percentage $=\frac{n \text { incorporated } \text { Chol }}{n \text { incorporated } D P P C} \times 100$

With: "n incorporated Chol" and "n incorporated DPPC" corresponding to the number of moles of Chol and DPPC incorporated in the liposome formulation respectively.

\subsection{Membrane permeability study}

\subsubsection{Purification of liposomal suspensions by molecular sieve chromatography}

The SRB-loaded liposomes were subjected to a centrifugation for 2 hours, at a speed of 15000 $\mathrm{rpm}$, at $4^{\circ} \mathrm{C}$. The pellets were suspended in $0,1 \mathrm{M}$ Tris- $\mathrm{HCl}$ buffer $(\mathrm{pH} \mathrm{7,4)} \mathrm{containing} 150 \mathrm{mM}$ $\mathrm{NaCl}$. This preliminary step facilitates the purification of liposomes on the $\mathrm{G} 25$ column by eliminating the excess buffer and allows a non-delicate separation of non-encapsulated molecules (free SRB, phospholipids and cholesterol) that will remain in the supernatant.

SRB-loaded liposomes were then separated from free SRB, phospholipids and cholesterol molecules by molecular sieve chromatography (using a Sephadex G25 column). The liposomal solution of each formulation was eluted with $0,1 \mathrm{M}$ Tris- $\mathrm{HCl}$ buffer $(\mathrm{pH} 7,4)$ containing $150 \mathrm{mM}$ $\mathrm{NaCl}$. The liposomes encapsulating SRB were first eluted whereas free molecules of SRB, DPPC and cholesterol were longer delayed by the gel and tardily eluted. The liposomal suspensions first eluted will be used for the membrane permeability study.

\subsubsection{Exposure of liposomal suspensions to EO components}

Liposomal suspensions of each preparation were diluted in a Tris $\mathrm{HCl}$ buffer containing $150 \mathrm{mM}$ $\mathrm{NaCl}(0.1 \mathrm{M}, \mathrm{pH}=7.4)$ in order to obtain liposomal solutions with an optical density of 0.5 at 535 $\mathrm{nm}$. The EOs components solutions prepared in methanol were then added to liposomes at a molar ratio DPPC:EO of (100: 25) and samples were incubated at $37^{\circ} \mathrm{C}$ for 72 hours. For each formulation, a liposomal solution treated with the same volume of methanol was used as a control and will be referred to as "blank liposomes". The corresponding EO volume added to the liposomes did not exceed $5 \%$ of the liposomal suspension volume. At this percentage, the methanol doesn't disrupt the liposomes stability (data not shown). 


\subsubsection{Membrane permeability study by fluorescence spectroscopy}

Membrane permeability was monitored by means of fluorophore leakage from vesicles. In fact, the loading of the SRB (in the liposomes aqueous core) at a high concentration generates a fluorescence quenching phenomenon due to the formation of non-fluorescent aggregates (Nasr et al., 2020). Dye release from liposomes leads to its dilution and subsequently, the restitution of the fluorescence. Therefore, the effect of the different EOs components tested in our study on membrane permeability was followed by measuring the increase of the fluorescent signal resulting from the release of $\mathrm{SRB}$ from liposomes with time at $37^{\circ} \mathrm{C}$.

The SRB loaded liposomes suspensions of each formulation were exposed or not to EOs components and incubated in a water bath at $37^{\circ} \mathrm{C}$. Aliquots were removed from each sample at 0 , 24, 48 and $72 \mathrm{~h}$, diluted 50 times in $0.1 \mathrm{M}$ Tris- $\mathrm{HCl}$ buffer $(\mathrm{pH} 7.4)$ containing $150 \mathrm{mM} \mathrm{NaCl}$ and the fluorescence signal was measured immediately.

To eliminate an eventual interference of EO components in fluorescence measurements, EO components were dissolved in a Tris $\mathrm{HCl} \mathrm{NaCl}(0.1 \mathrm{M}, \mathrm{pH} 7.4)$ buffer at the same concentration used for the liposomal permeability study.

Fluorescence measurements were performed using a spectrofluorometer (Hitashi F-7000 Spectrofluorometer) at an excitation wavelength of $535 \mathrm{~nm}$ and an emission wavelength of 590 $\mathrm{nm}$. The emission spectrum was recorded in the range 540-700 $\mathrm{nm}$. The permeability of liposomal membranes was evaluated by calculating the percentage of the fluorophore released from LUVs versus time, using the following equation:

SRB release percentage $=\frac{(F t-F 0)}{F \max } \times 100$

Where Ft is the fluorescence intensity measured at time $t, F_{0}$ is the fluorescence intensity measured at time 0 and Fmax is the maximum fluorescence indicating a complete release of SRB from vesicles and obtained in the presence of the nonionic detergent, Triton X-100 (1\%) in a Tris $\mathrm{HCl}$ buffer (0.1 M ; pH 7.4) containing $150 \mathrm{mM} \mathrm{NaCl}$. 
The effect of an EO component on the permeability of liposomal membrane of each formulation was determined as followed:

Permeabilizing effect of an $E O=\frac{S R B \text { release } \% \text { from liposomes } E O S}{S R B \text { release } \% \text { of blank liposomes }}$

Where the "SRB release \% from liposomes EOs" corresponds to the SRB release percentage of liposomes samples treated with EO components and the "SRB release \% of blank liposomes" is the SRB release percentage of the control.

\subsection{Statistical analysis}

To assess significant differences between values, statistical analysis was carried out using the Student's t-test. A value of $\mathrm{P}<0.05$ was considered statistically significant.

\section{Results and Discussion}

\subsection{Characterization of blank liposomes}

\subsubsection{Size analysis}

All the liposomal batches underwent an extrusion through polycarbonate membranes of $400 \mathrm{~nm}$ pore size, expecting thereafter a theoretical vesicle mean diameter equal to this size. Our results were in accordance with previously published data carried out in our laboratory where the mean size of DPPC/Chol formulations gradually increased following the addition of Chol to the membrane up to $30 \%$ Chol. No further increase in the vesicles size was observed at high Chol content (50\% and $100 \%$ Chol membranes) since liposomal membranes had a mean diameter close to $400 \mathrm{~nm}$ regardless of increasing Chol content (data not shown) (Kaddah et al., 2018).

\subsubsection{Phospholipids and Cholesterol quantification of blank LUVs}

Total and free DPPC and Chol were determined for each formulation as described above. The incorporation rates and the loading rates were calculated according to equations (1), (2), (3) and (4). The DPPC:Chol molar ratio determined experimentally in the final liposomal membranes are presented in table 1 .

Table 1: DPPC and Chol loading rates and incorporation rates for each liposomal formulation (DPPC:Chol molar ratios: 100:10; 100:25; 100:50; 100:75 and 100:100). The corresponding DPPC 
and Chol mole number as well as the experimentally determined DPPC:Chol molar ratio are also presented for each formulation. Values are expressed as the means of three measurements \pm SD.

\begin{tabular}{|c|c|c|c|c|c|}
\hline \multirow{2}{*}{$\begin{array}{c}\text { Theoretical } \\
\text { DPPC:Chol } \\
\text { molar ratio }\end{array}$} & LR (\%) & IR (\%) & LR (\%) & IR (\%) & $\begin{array}{c}\text { Experimentally } \\
\text { determined } \\
\text { DPPC:Chol ratio }\end{array}$ \\
\hline $100: 10$ & $85.86 \pm 0.008$ & $98.9 \pm 0.007$ & $100.00 \pm 0.05$ & $100.00 \pm 0.41$ & $100: 11$ \\
\hline $100: 25$ & $77.22 \pm 0.005$ & $98.53 \pm 0.06$ & $65.98 \pm 1.47$ & $83.41 \pm 0.89$ & $100: 21$ \\
\hline $100: 50$ & $76.2 \pm 0.001$ & $97.83 \pm 0.004$ & $60.43 \pm 0.62$ & $92.35 \pm 0.92$ & $100: 40$ \\
\hline $100: 75$ & $75.36 \pm 0.009$ & $98.76 \pm 0.004$ & $43.58 \pm 1.15$ & $98.30 \pm 1.25$ & $100: 43$ \\
\hline $100: 100$ & $77.6 \pm 0.015$ & $95.67 \pm 0.002$ & $42.55 \pm 1.72$ & $97.54 \pm 0.20$ & $100: 55$ \\
\hline
\end{tabular}

We found that the DPPC loading rates were around 75\% for all liposomal batches except for 10\% Chol membrane where a greater loading rate value $(85.86 \%)$ was obtained. These values show that the Chol content influences the incorporation of DPPC into the membrane: in fact, Chol reduces DPPC incorporation up to $25 \%$ Chol content (the LR of DPPC decreased from $85.86 \%$ for $10 \%$ Chol membranes to $77.22 \%$ for $25 \%$ Chol membranes). Above this concentration, no influence of increasing Chol membrane content on the incorporation of DPPC is observed (the LR of DPPC ranged from 75 to $77 \%$ for all formulations).

On the other hand, the DPPC and Chol incorporation rates of all liposomal batches were high proving consequently the efficacy of the reverse evaporation phase method chosen for the preparation of liposomes. We noticed also that, at $10 \% \mathrm{Chol}$, all the Chol molecules were incorporated in the membrane (LR value was of $100 \%$ ). With increasing Chol content, the loading rates of Chol gradually decreased reaching $42.5 \%$ for the formulation of DPPC:Chol (100:100). Indeed, at low Chol content (up to 25\%), the theoretical DPPC:Chol molar ratio is practically respected since the experimentally determined molar ratios were nearly the same as predicted. Conversely, at high Chol content (50\% Chol and more), the experimentally determined DPPC:Chol molar ratios did not reflect anymore the theoretical ratios: at 50\% Chol, the liposome membranes were found to contain $40 \%$ Chol. Similarly, DPPC membranes theoretically incorporating $75 \%$ and $100 \%$ Chol were actually composed of $43 \%$ and $55 \%$ Chol respectively. Our results are in agreement with (Ibarguren et al., 2010), who quantified the Chol content of liposome membranes made of different lipid binary mixtures before and after extrusion. In most 
cases, the extrusion resulted in liposomes with Chol contents lower than the theoretical ratios: particularly, the extruded DPPC/Chol membranes with Chol molar ratios ranging from 33\% to $75 \%$ have shown Chol incorporation rates below the predicted values. This effect was pronounced with increasing Chol content. A possible explanation for this is that the Chol molecules are kicked out of the membrane and remain stuck on the filters during the extrusion process. One can also note here that the effective composition of liposome formulations containing $50 \%$ and $75 \%$ Chol is nearly the same (40\% and $43 \%$ Chol respectively) expecting no significant difference between these 2 formulations. Notwithstanding, the permeability study results revealed different behaviors

of EO compounds on these membranes. Thus, this difference appears to be significant and could not be neglected.

\subsection{Permeability study: SRB release from liposomes}

The liposomal membrane permeability was assessed by the commonly used fluorescence technique: vesicle leakage of self-quenching probes (Nasr et al., 2020). The LUVs encapsulating the fluorescent agent SRB were exposed to EOs components at a molar ratio DPPC:EO of 100:25. The choice of this ratio was based on a biophysical study conducted by (Gharib et al., 2018) where the effect of a series of EO monoterpenes compounds on the fluidity of DPPC membrane was investigated at various molar percentages of EO components to DPPC (from 0 to $25 \mathrm{~mol} \%$ ) and in the absence of Chol. The studied molecules exerted a fluidizing effect on DPPC membranes, this effect being enhanced in a dose dependent manner. Considering these results, the effect of Chol on the membrane permeability (Kaddah et al., 2018) and our aim to find adequate formulations for EOs encapsulation, we selected the highest ratio (25 mol\%) expecting a high amount of the EO molecules retained in the liposome structures.

The permeability of LUVs was evaluated by measuring the fluorescence signal of liposomal suspensions at $37^{\circ} \mathrm{C}$ between $0 \mathrm{~h}$ and $72 \mathrm{~h}$. We should note that the SRB release from liposomes composed of DPPC and various Chol content was time dependent and showed a biphasic release pattern (Kaddah et al., 2018). It includes an initial fast SRB release during the first 8 to $10 \mathrm{~h}$, followed by a landing period (between 10 and $24 \mathrm{~h}$ ) where no considerable dye leakage is observed. With respect to these results, we have chosen to carry out the permeability study in the presence of EOs components during 72 hours to cover both phases of the SRB release kinetics.

\subsubsection{Fluorescence of EOs components}


None of the studied EO molecules emit a fluorescence signal in the range of 500-700 nm and, therefore, the fluorescence increase observed in the following experiments is due only to the leakage of SRB and thus the inhibition of fluorescence quenching rather than the molecules structure (figure 1, supplementary materials).

\subsubsection{SRB release from liposomal formulations of various Chol content}

According to (Kaddah et al., 2018), liposome membranes made of DPPC:Chol (100:10) was the less stable and the most permeable formulation. Its SRB release pattern was comparable to free Chol membranes. Therefore, this formulation was chosen to test the effect of a series of 22 EO components on membrane permeability. Table 2 represents the permeabilizing effect of EO components on DPPC:Chol (100:10) liposomes obtained after $72 \mathrm{~h}$ of incubation and calculated according to equation (7).

Table 2: The permeabilizing effect of EO components obtained with the DPPC:Chol (100:10) liposomes after $72 \mathrm{~h}$ of incubation at $37^{\circ} \mathrm{C}$. Fluorescence measurements were carried out on a spectrofluorometer at an excitation wavelength of $535 \mathrm{~nm}$ and an emission wavelength of $590 \mathrm{~nm}$.

Values are expressed as the means of three different measurements $\pm \mathrm{SD}$.

(* indicates a value of $\mathrm{P}<0.05$ statistically significant)

\begin{tabular}{|l|c|}
\hline \multicolumn{1}{|c|}{ Sample } & $\begin{array}{c}\text { SRB release ratio of sample } \\
\text { to that of blank at 72h }\end{array}$ \\
\hline Blank & 1.00 \\
\hline \multicolumn{2}{|c|}{ Phenolic compounds } \\
\hline Eugenol & $1.37 \pm 0.02^{*}$ \\
\hline Thymol & $1.30 \pm 0.11^{*}$ \\
\hline Guaiacol & $1.27 \pm 0.06^{*}$ \\
\hline trans-Anethole & $1.19 \pm 0.02^{*}$ \\
\hline Eugenyl acetate & $1.15 \pm 0.01^{*}$ \\
\hline Anisole & $1.04 \pm 0.12$ \\
\hline Estragole & $0.99 \pm 0.01$ \\
\hline Isoeugenol & $0.93 \pm 0.02^{*}$ \\
\hline & $1.43 \pm 0.05^{*}$ \\
\hline Nerolidol & $1.37 \pm 0.04^{*}$ \\
\hline Linalool & $1.26 \pm 0.08^{*}$ \\
\hline Geraniol & $1.23 \pm 0.06^{*}$ \\
\hline$\alpha-$ Phellandrene & $1.19 \pm 0.02^{*}$ \\
\hline$\alpha$-Terpinene & $1.18 \pm 0.05^{*}$ \\
\hline Linalyl acetate & $1.17 \pm 0.01^{*}$ \\
\hline Menthol & \\
\hline
\end{tabular}




\begin{tabular}{|l|c|}
$\gamma$-Terpinene & $1.17 \pm 0.06^{*}$ \\
\hline Bornyl acetate & $1.05 \pm 0.08$ \\
\hline Camphor & $1.03 \pm 0.02$ \\
\hline$\beta$-Caryophyllene & $1.02 \pm 0.04$ \\
\hline Camphene & $0.98 \pm 0.05$ \\
\hline Borneol & $0.91 \pm 0.005^{*}$ \\
\hline p-Cymene & $0.86 \pm 0.07^{*}$ \\
\hline
\end{tabular}

Based on the statistical significance t-test, among the 22 EO components tested, only 13 compounds increased the permeability of DPPC:Chol (100:10) liposomes after 72 hours of incubation since their permeabilizing effects were significantly higher than 1 (release value of blank liposomes). In a descending order of permeabilizing effect, the 13 selected molecules are classified as follows: nerolidol $>$ eugenol $>$ linalool $>$ thymol $>$ guaiacol $>$ geraniol $>\alpha$ phellandrene $>$ trans-anethole $>\alpha$-terpinene $>$ linalyl acetate $>$ menthol $>\gamma$-terpinene $>$ eugenyl acetate. At this DPPC/EO molar ratio, the remaining components (anisole, estragole, isoeugenol, bornyl acetate, camphor, $\beta$-caryophyllene, camphene, borneol and p-cymene) had no significant effect on the liposomal permeability suggesting that another mechanism of action may be accountable for their antimicrobial activities. Interestingly, we can notice that three EO components (isoeugenol: $0.93 \pm 0.02$, borneol: $0.91 \pm 0.005$ and p-cymene: $0.91 \pm 0.005$ ) seem to exert a stabilizing effect on the liposome formulation since their SRB release values were significantly below that of the blank.

Our results showed that, among the 13 EO components previously mentioned, the majority were terpenic compounds bearing an alcohol function (phenolic compounds: eugenol, thymol and guaiacol and non-phenolic compounds: nerolidol, linalool, geraniol, menthol). In fact, numerous studies highlighted the importance of the hydroxyl group for the biological activity of several phenolic compounds exhibiting a permeabilizing effect (Burt, 2004; Ultee et al., 2002). Among the most studied phenylpropanoids, eugenol was proved to permeabilize the bacterial cell membrane (Hyldgaard et al., 2012). Thymol, another OH-bearing compound, was demonstrated to interact with the cytoplasmic cell membrane and integrate into the membrane via its hydroxyl group at the polar head groups of the lipid bilayer leading to the loss of membrane integrity and to the increase in its passive permeability (Helander et al., 1998; Walsh et al., 2003; Radulovic et al., 2013). Accordingly, in our study, eugenol and thymol showed a permeabilizing effect of $1.37 \pm$ 
0.02 and $1.30 \pm 0.11$ respectively. In addition, guaiacol which has common structural features with eugenol (both possess a hydroxyl and a methoxy groups) but lacks the prop-2-enyl side chain, was also able to permeabilize liposome membranes containing $10 \%$ Chol $(1.27 \pm 0.06)$. This suggests that the prop-2-enyl chain may help the molecule to better interact with or fit into the membrane. On the other hand, the substitution of the hydroxyl group (case of eugenol) with an acetate group in eugenyl acetate reduced the effect of this latter on the membrane. Similarly, trans-anethole which has no hydroxyl group has shown a weak permeabilizing effect comparable to that of eugenyl acetate $(1.19 \pm 0.02$ and $1.15 \pm 0.01$ for trans-anethole and eugenyl acetate respectively). This holds true when comparing the effect of guaiacol and anisole. Lacking the alcohol function, anisole did not show any effect on the membrane. This could be also the reason for which estragole does not affect the vesicle permeability. These instances further underline the importance of $\mathrm{OH}$ and its direct implication in the EO-membrane permeability.

Beside the importance of this $\mathrm{OH}$ group, our results revealed the presence of other structural features that could influence the membrane permeability. Isoeugenol, the isomer of eugenol, showed no permeabilizing effect on liposomes made from the binary mixture of DPPC:Chol (100:10); it rather displayed a stabilizing effect on the membrane $(0.93 \pm 0.02)$. Indeed, the double bond in the $\alpha, \beta$ positions and the methyl group in the $\gamma$ position of isoeugenol's side chain create a conjugated system which is absent in the eugenol's structure. This structural feature appears to upset the permeabilizing effect of isoeugenol and to convert the eugenol induced permeability $(1.37 \pm 0.02)$ into a stabilizing effect with isoeugenol $(0.93 \pm 0.02)$. Therefore, we can suggest that the interaction of EO components with phospholipid membranes does not only account for the presence of a hydroxyl group. However, isoeugenol is known for its antibacterial activity against various bacterial strains (Zhang et al., 2017) and was also shown to induce the leakage of calceinloaded LUVs made from E. Coli lipid extract (Hyldgaard et al., 2015). Moreover, it demonstrated a deleterious effect on liposome membrane stability based on DSC results (Gharib et al., 2017). These discrepancies might be due to the presence of cholesterol in the present formulations comparing to other studies where cholesterol was absent.

Regarding the non-phenolic compounds, the importance of the hydroxyl group was also emphasized: a permeabilizing effect was obtained for $\mathrm{OH}$-bearing molecules, with nerolidol (a linear sesquiterpene) exhibiting the highest effect $(1.43 \pm 0.05)$. Interestingly, the linear terpenes 
(nerolidol, linalool and geraniol) showed a better effect on $10 \%$ Chol membranes compared to the cyclic terpene, menthol, whose effect was similar to that of hydrocarbon monoterpenes (nerolidol: $1.43 \pm 0.05$, linalool: $1.37 \pm 0.04$ and geraniol: $1.26 \pm 0.08$ versus menthol: $1.17 \pm 0.01, \alpha-$ phellandrene: $1.23 \pm 0.06, \alpha$-terpinene: $1.19 \pm 0.02, \gamma$-terpinene: $1.17 \pm 0.06)$. Here also, the replacement of the hydroxyl group of linalool with an acetate group reduced the effect of linalyl acetate $(1.37 \pm 0.04$ and $1.18 \pm 0.05$ for linalool and linalyl acetate respectively). Although bearing an alcohol function, borneol $(0.91 \pm 0.005)$ was found to reduce the permeability of liposome membrane. The bulk structure of this bicyclic monoterpene could modulate its interaction with liposome membrane, and consequently its permeabilization effect. Moreover, as regards the molecules lacking $\mathrm{OH}$, the presence of a conjugated system in p-cymene reversed the permeabilizing effect of $\alpha$-phellandrene and promoted a more stable membrane $(0.86 \pm 0.07$ and $1.23 \pm 0.06$ for $\mathrm{p}$-cymene and $\alpha$-phellandrene respectively). Besides the presence of a conjugated system, the hydrophobicity of p-cymene ( $\log$ P 4.02) can further explain its stabilizing effect for this molecule would be much retained inside the membrane.

Overall, it could be concluded that the presence of a hydroxyl group, the presence of a conjugated system and the linearity of the molecule are important structural features modulating membrane permeability.

Based on the above preliminary results, liposomes of different Chol content were treated with only the EO compounds that showed a permeabilizing effect at $10 \%$ Chol (13 compounds). Figures 2 and 3 represent the permeabilizing effect of phenolic and non-phenolic EO components with all liposomal formulations (DPPC:Chol 100:10; 100:21; 100:40; 100:43 and 100:55 molar ratios) obtained after 72 hours of incubation at $37^{\circ} \mathrm{C}$. 


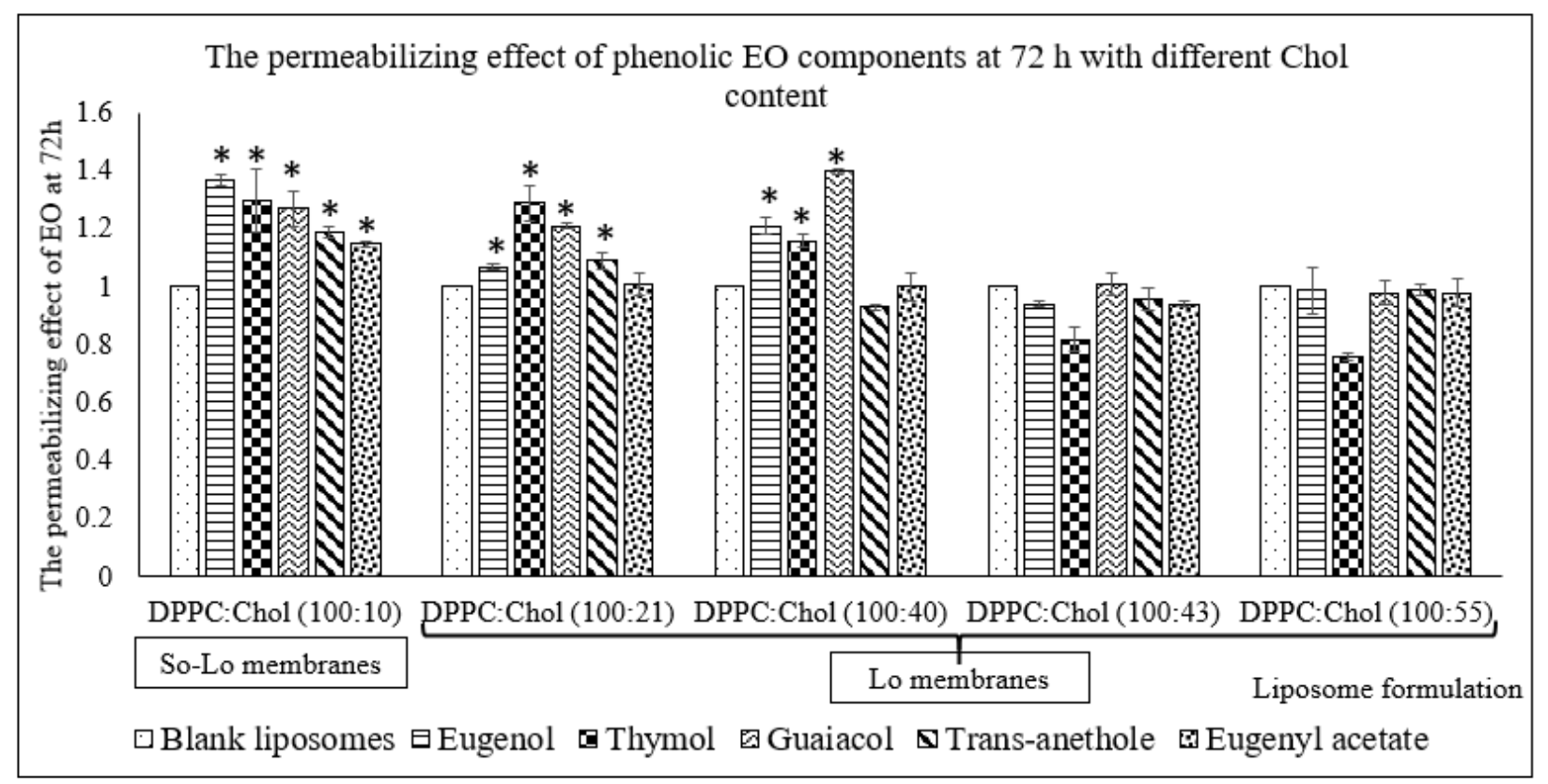

Figure 2: The permeabilizing effect of phenolic EO components with the different liposomal formulations (DPPC:Chol 100:10; 100:21 ; 100:40;100:43; 100:55) at 72h. (* indicates a value of $\mathrm{P}<0.05$ statistically significant)

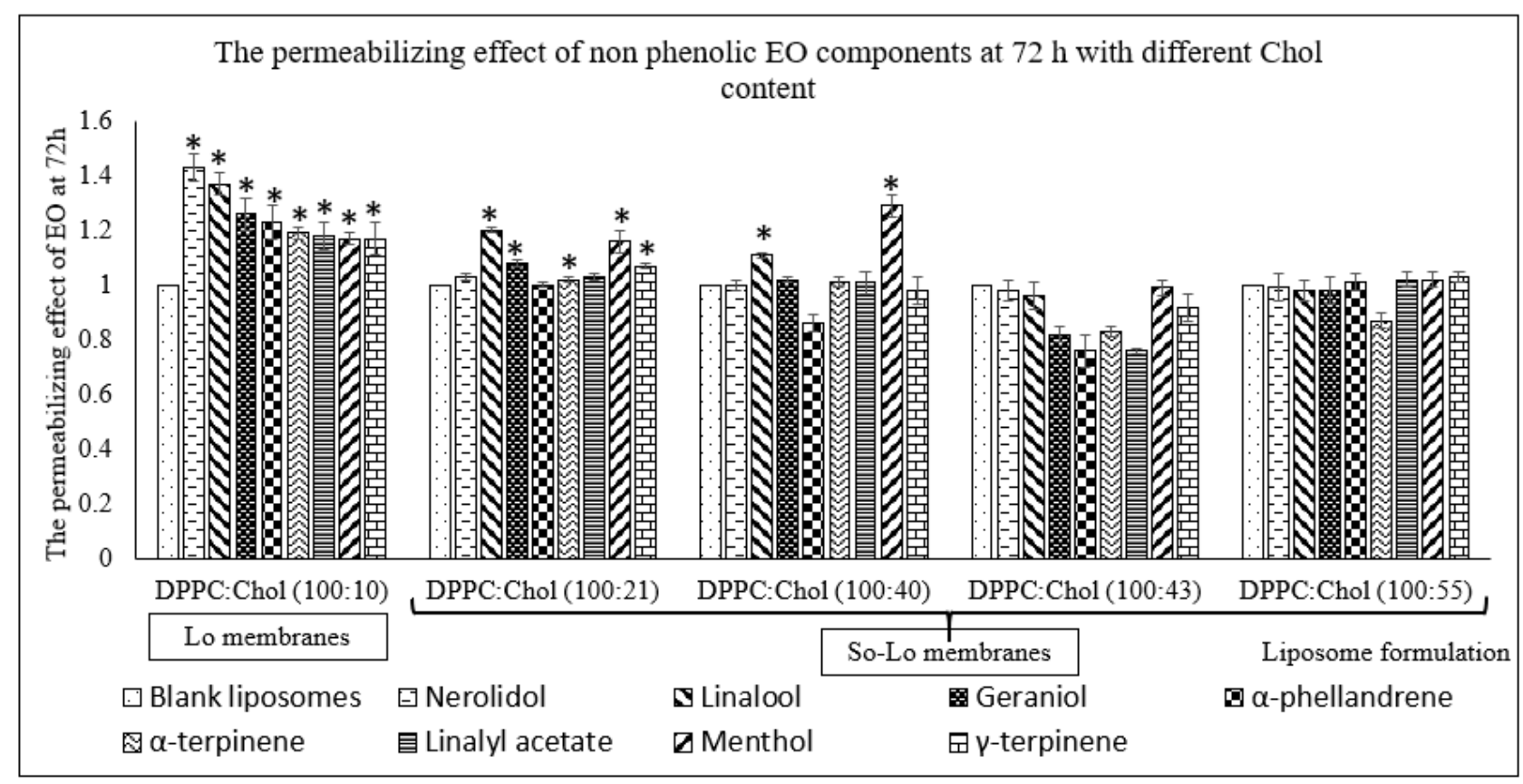

Figure 3: The permeabilizing effect of non-phenolic EO components with the different liposomal formulations (DPPC:Chol 100:10; 100:21; 100:40;100:43;100:55) at 72h. (* indicates a value of $\mathrm{P}<0.05$ statistically significant) 
It is quite obvious how Chol drastically affected the membrane permeability induced by the tested molecules. As a matter of fact, upon increasing the Chol content in liposomes, the permeabilizing effect of these compounds started to fade and, in some cases, to disappear. Effectively, results show that, among the $13 \mathrm{EO}$ components exerting a permeabilizing effect on liposomes containing $10 \%$ Chol, only 6 molecules bearing an $\mathrm{OH}$ group (eugenol, guaiacol, thymol, geraniol, linalool, menthol) along with the phenylpropene trans-anethole and two hydrocarbons monoterpenes ( $\alpha$ and $\gamma$-terpinene) kept a permeabilizing effect on liposomes composed of $21 \%$ Chol. The effect of eugenol, guaiacol, thymol, linalool and menthol remained up to $40 \%$ Chol. As for liposomes composed of $43 \%$ and $55 \% \mathrm{Chol}$, none of the tested molecules showed a significant permeabilizing effect on the lipid bilayer. Although the slight difference in the experimentally determined Chol content between the $40 \%$ and $43 \%$ Chol membranes (50 and $75 \%$ Chol), the behavior of EO components towards these 2 formulations was not the same: our findings indicate that the permeabilizing effect of EO components can be observed up to $40 \%$ Chol membranes. Above this percentage, their effect is inhibited. For instance, guaiacol and eugenol showed a permeabilizing effect of $1.40 \pm 0.01$ and $1.21 \pm 0.03$ on $40 \%$ Chol membranes respectively. Their effects were significantly reduced at $43 \% \mathrm{Chol}$ membranes to reach $1.01 \pm 0.04$ and $0.94 \pm 0.01$ respectively. The same could be observed with linalool, menthol, thymol, etc.

The hydrophobicity of the compound is a key parameter that could affect the interaction of EO constituents with the membrane. Our results showed that when the EO component is highly hydrophobic ( $\log \mathrm{P}>4$ ), two scenarios could take place depending on the structure of the molecule: in the presence of a hydroxyl group, a permeabilizing effect occurs only at low Chol content. This is the case of nerolidol, a hydrophobic compound ( $\log \mathrm{P} 4.6)$, which only permeabilized DPPC/Chol membranes made of $10 \%$ Chol. Conversely, when the hydrophobic molecule lacks a hydroxyl group, no permeabilizing effect is seen. For example, p-cymene (log P 4.02) and $\beta$ caryophyllene ( $\log$ P 4.4), highly hydrophobic compounds with no alcohol function, did not enhance the permeability of any formulation. Furthermore, it was previously stated that at high Chol content, Chol reduces the drug-membrane interaction because a competition might occur between EO compounds and Chol in the membrane (Sherry et al., 2013). For this reason, the permeabilizing effect of nerolidol might be inhibited at higher Chol content. 
The decrease in the hydrophobicity of EO components possessing a $\mathrm{OH}$ group will increase the extent of membrane permeabilization at high Chol content: actually, the EO components bearing a hydroxyl group with $\log \mathrm{P}$ below 4 , had a permeabilizing effect visible up to $40 \%$ Chol. However, geraniol ( $\log$ P 2.9) with similar hydrophobicity to linalool (log P 2.7) exerted a weaker permeabilizing effect only up to $21 \%$ Chol membranes (linalool: $1.37 \pm 0.04 ; 1.20 \pm 0.01 ; 1.11 \pm$ 0.01 versus geraniol: $1.26 \pm 0.06 ; 1.08 \pm 0.01 ; 1.02 \pm 0.01$ at $10 \%, 21 \%$ and $40 \%$ Chol respectively). This could be probably explained by the differential position of the hydroxyl group in each compound: the $\mathrm{OH}$ group of geraniol is at the end side of the molecule which make the structure less disturbing when it reaches the membrane comparing to geraniol.

Regarding EO components having no hydroxyl group and with a $\log$ P lower than 4, a very weak permeabilizing effect persisted for $\alpha$-terpinene $(\log$ P 2.8), $\gamma$-terpinene $(\log$ P 2.8) and transanethole $(\log$ P 3.3$)$ up to $21 \%$. The remaining molecules in this category had no significant effect regardless the Chol content. All these results are summarized in table 2, supplementary materials.

On the other hand, lipid bilayers could exist in different states depending on many parameters; particularly, Chol concentrations and temperature (Vist and Davis, 1990; McMullen and McElhaney, 1995). In our study, membrane permeability was assessed by following SRB release from liposomes at $37^{\circ} \mathrm{C}$ which corresponds to a temperature below the melting temperature of DPPC $\left(41^{\circ} \mathrm{C}\right)$. Therefore, membrane organization of the liposomes is controlled by Chol content. Based on the work of Sankararn and Thompson (1991), the liposomal formulations exist in two different membrane states. For DPPC membranes with Chol concentration ranging from 7 to 30 mol\% and below Tm, the lipid bilayer is in the solid ordered - liquid ordered phase (So-Lo) in which both gel and liquid-ordered phases coexist. As for membranes with Chol concentrations above $30 \mathrm{~mol} \%$, lipid bilayers adopt the liquid-ordered phase (Lo) (Sankaram and Thompson; 1991). Our results showed that in the (So-Lo) transition phase (membranes composed of $10 \%$ and $21 \%$ Chol effective rates), the permeabilizing effect of EOs was pronounced at $10 \%$ and then decreased or disappeared by increasing cholesterol content (table 3, supplementary materials). In the Lo phase (for membranes effectively composed of $40 \%, 43 \%$ and 55\% Chol), five EOs components possessing a hydroxyl group kept their permeabilizing effect (menthol, eugenol guaiacol, thymol and linalool) on 40\% Chol membranes only. This underscores again the importance of $\mathrm{OH}$ group in the EO induced permeability and highlights as well the stability 
conferred by Chol to the membrane. In fact, the addition of increasing Chol concentrations implies the transition from a leaky to a more stable membrane leading to a decrease in the membrane permeability of liposomes as demonstrated by (Kaddah et al., 2018). Therefore, the introduction of Chol into the lipid bilayer results in a membrane that resists the disturbance and destabilization caused by bioactive agents as we obtained with the thymol, where the increase in Chol content results in a decrease of SRB release ratios from liposomes. (table 3, supplementary materials or figure 4a). Unexpectedly, for eugenol, guaiacol and menthol there was a re-increase in the permeabilizing effect in the Lo phase at $40 \%$ Chol content followed by a dramatical decrease at $43 \%$ where no permeabilizing effect was observed (table 3, supplementary materials or figure 4 b). A plausible explanation to this observation could be the heterogeneity of the membrane at high Chol content. Effectively, the incorporation of large amount of Chol will give rise to lipid rafts, rich-Chol nanodomains, alongside with poor-Chol domains generating thereby an heterogenous membrane (Lopez-Pinto et al., 2005; Tseng et al., 2007). Hence, this membrane contains at the same time domains with high physical stability (rich-Chol domains) and domains with low physical stability (poor-Chol domains). The EO components will probably tend to permeabilize these unstable domains. This would account for the re-increase in the permeabilizing effect of some compounds at $40 \%$ or $43 \%$ Chol.

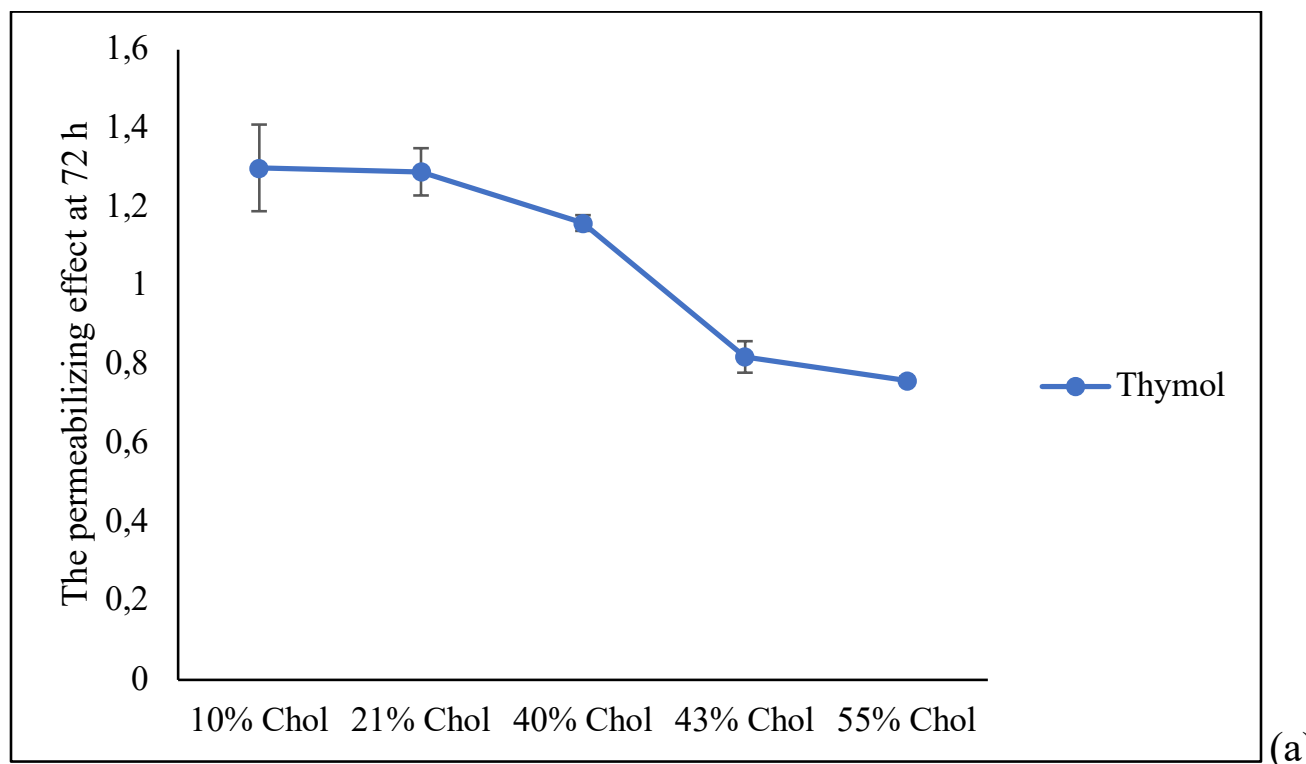




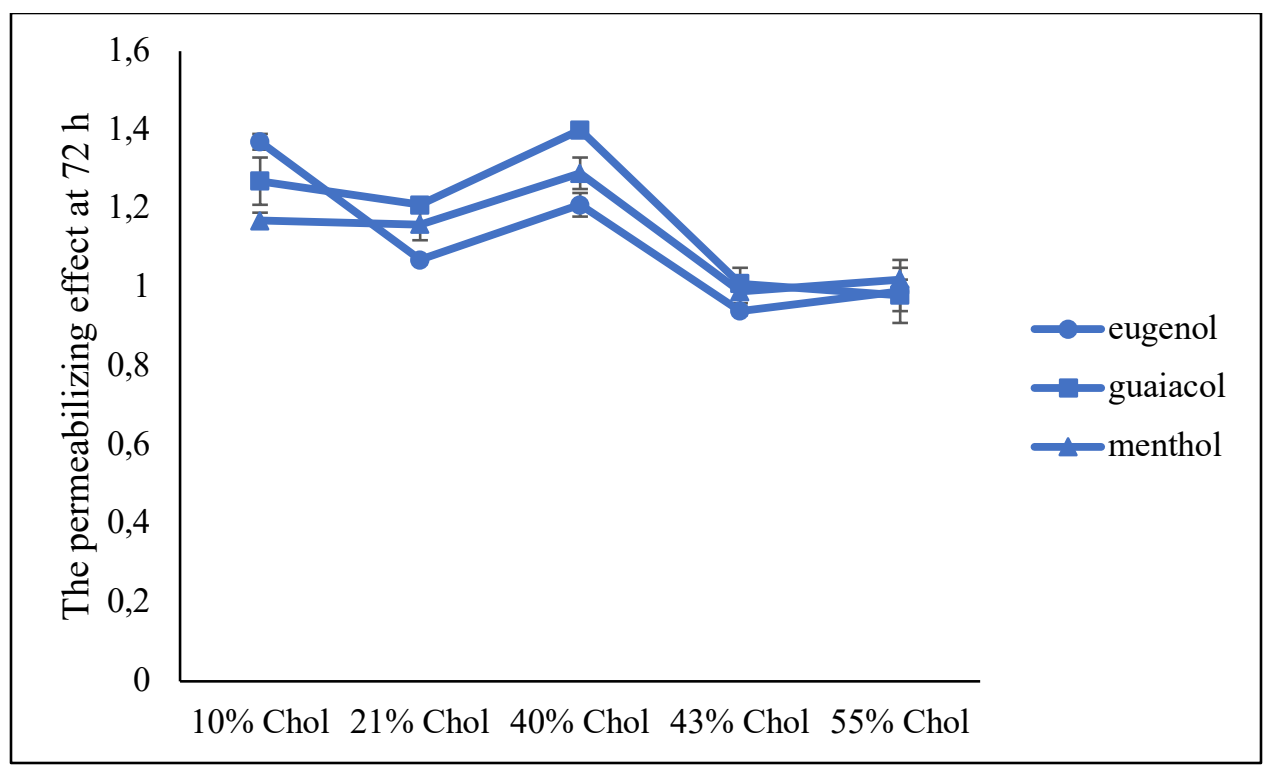

(b)

Figure 4: The permeabilizing effect of EO components obtained at $72 \mathrm{~h}$ with various Chol content. Two patterns were obtained: a decreasing effect with increasing Chol content depicted in figure 4 (a) for thymol, a re-increase in EO permeabilizing effect at 50\% Chol shown in figure 4(b) for three components: eugenol, guaiacol and menthol.

Taking in consideration all the discussed parameters, we can assume that the EOs effect on membrane permeability in the presence of Chol can be modulated by the structure of the molecule (presence of hydroxy group, position of the double bound, linearity of the molecule, length of the hydrocarbon chain), its hydrophobicity and the state of the membrane. Therefore, all these parameters can help us choose the best liposome formulation for the EOs encapsulation. As discussed above, the hydroxyl group in the EOs seems to be linked to the membrane permeability; however, the hydrophobicity of the components should be taken into account when choosing the more stable encapsulation formulation. For instance, nerolidol, bearing an $\mathrm{OH}$ group and possessing a $\log \mathrm{P}$ higher than 4 , permeabilized the membrane up to $10 \%$ Chol. Nevertheless, due to its high hydrophobicity, a competition with the Chol may occur at high Chol content resulting in a low encapsulation efficiency as obtained for many hydrophobic drugs with liposomal formulations rich in Chol (Jaafar-Maalej et al., 2010; Sherry et al., 2013; Varona et al., 2011). Thus, a formulation moderately rich in Chol (not exceeding 40\%) has to be chosen for the encapsulation of the compound. On the other side, linalool, bearing an $\mathrm{OH}$ group and possessing a low hydrophobicity permeabilized the membrane up to $40 \%$ Chol. Therefore, a formulation with a Chol content above $40 \%$ is recommended for a stable encapsulation. Consequently, a liposomal 
formulation loaded with either eugenol, thymol, guaiacol or menthol should be also composed of a Chol concentration higher than $40 \%$. Considering the remaining non-phenolic compounds with no hydroxyl group and possessing a permeabilizing effect on the permeability of liposome membranes, we should note that these components were active at low Chol contents (10 and 21\% Chol). These components must be encapsulated in a formulation moderately rich in Chol for most of these compounds are hydrophobic.

\section{Conclusions}

In this work, we investigated the effect of a series of $22 \mathrm{EO}$ components belonging to different classes (phenylpropenes, oxygenated monoterpenes and sesquiterpenes) on the permeability of DPPC liposome membranes with various Chol content $(10 ; 21 ; 40 ; 43$ and 55\% Chol). Our results helped us to establish a correlation between the structure of a compound, its hydrophobicity, the membrane state and its effect on the membrane permeability.

EO constituents bearing a hydroxyl group have demonstrated the best permeabilizing effect; this effect being reduced with increasing Chol content in the So-Lo phase. Upon increasing Chol content, in the liquid-ordered phase, a re-increase in the permeabilizing effect of some EO components could be attributed to the heterogeneity of the membrane. The permeabilizing effect of EO components was visible up to $40 \%$ Chol only; none of the studied molecules displayed a significant effect at higher Chol content. Hydrophobicity of the compound played also a crucial role. Hydrophobic compounds having no hydroxyl group in their structure showed a low permeabilizing effect only in the So-Lo phase. No significant effect on the membrane was observed in the liquid-ordered phase. As for hydrophobic compounds possessing a hydroxyl group, when the hydrophobicity of EO component increases the Chol content up to which a permeabilizing effect is observed will decrease. Particularly in this category, the highly hydrophobic compound nerolidol displayed a significant permeabilizing effect at $10 \%$ Chol. This effect disappeared at higher content.

Accordingly, Cholesterol modulates not only the membrane permeability but also the interactions that may occur between the hydrophobic compounds and the membrane lipids. Therefore, increasing the Chol to phospholipid ratio to some extent wouldn't be useful for the encapsulation of highly hydrophobic compounds since Chol will reduce their encapsulation efficiency at high 
Chol concentrations. That's why, the DPPC:Chol ratio of the formulation is selected depending on the structure of the compound, its hydrophobicity and its consequent effect on the permeability at different membrane states. Based on the above considerations, we can conclude that a liposome formulation with Chol not exceeding $40 \%$ would be suitable for the encapsulation of highly hydrophobic molecules whereas a formulation comprising higher Chol content (above $40 \%$ ) could be suggested for less hydrophobic compounds. Finally, the Chol to EO component ratio is also a crucial parameter that has to be carefully chosen to circumvent the problems associated with low encapsulation of EOs in liposomes. Further investigations should be undertaken in order to determine the appropriate molar ratio of the ternary mixture: DPPC, Chol and EO component resulting in a stable formulation for EO encapsulation. 


\section{References}

Abu Lila, A.S., Ishida, T., 2017. Liposomal Delivery Systems: Design Optimization and Current Applications. Biol. Pharm. Bull. 40, 1-10. https://doi.org/10.1248/bpb.b16-00624

Asbahani, A.E., Miladi, K., Badri, W., Sala, M., Addi, E.H.A., Casabianca, H., Mousadik, A.E., Hartmann, D., Jilale, A., Renaud, F.N.R., Elaissari, A., 2015. Essential oils: From extraction to encapsulation. Int. J. Pharm. 483, 220-243. https://doi.org/10.1016/j.ijpharm.2014.12.069

Astani, A., Reichling, J., Schnitzler, P., 2011. Screening for Antiviral Activities of Isolated Compounds from Essential Oils. Evid. Based Complement. Alternat. Med. 2011, 1-8. https://doi.org/10.1093/ecam/nep187

Azzi, J., Jraij, A., Auezova, L., Fourmentin, S., Greige-Gerges, H., 2018. Novel findings for quercetin encapsulation and preservation with cyclodextrins, liposomes, and drug-incyclodextrin-in-liposomes. $\quad$ Food Hydrocoll. 81, 328-340. https://doi.org/10.1016/j.foodhyd.2018.03.006

Bakkali, F., Averbeck, S., Averbeck, D., Idaomar, M., 2008. Biological effects of essential oils A review. Food Chem. Toxicol. 46, 446-475. https://doi.org/10.1016/j.fct.2007.09.106

Boukhris, M., Bouaziz, M., Feki, I., Jemai, H., El Feki, A., Sayadi, S., 2012. Hypoglycemic and antioxidant effects of leaf essential oil of Pelargonium graveolens L'Hér. in alloxan induced diabetic rats. Lipids Health Dis. 11, 81. https://doi.org/10.1186/1476-511X-11-81

Bozzuto, G., Molinari, A., 2015. Liposomes as nanomedical devices. Int. J. Nanomedicine 975. https://doi.org/10.2147/IJN.S68861

Burt, S., 2004. Essential oils: their antibacterial properties and potential applications in foods-a review. Int. J. Food Microbiol. 94, 223-253. https://doi.org/10.1016/j.ijfoodmicro.2004.03.022

Chen, Y., Zeng, H., Tian, J., Ban, X., Ma, B., Wang, Y., 2013. Antifungal mechanism of essential oil from Anethum graveolens seeds against Candida albicans. J. Med. Microbiol. 62, 11751183. https://doi.org/10.1099/jmm.0.055467-0

Chung, M.J., Cho, S.-Y., Bhuiyan, M.J.H., Kim, K.H., Lee, S.-J., 2010. Anti-diabetic effects of lemon balm ( Melissa officinalis) essential oil on glucose- and lipid-regulating enzymes in type 2 diabetic mice. Br. J. Nutr. 104, 180-188. https://doi.org/10.1017/S0007114510001765

Cristani, M., D’Arrigo, M., Mandalari, G., Castelli, F., Sarpietro, M.G., Micieli, D., Venuti, V., Bisignano, G., Saija, A., Trombetta, D., 2007. Interaction of Four Monoterpenes Contained in Essential Oils with Model Membranes: Implications for Their Antibacterial Activity. J. Agric. Food Chem. 55, 6300-6308. https://doi.org/10.1021/jf070094x

Cui, H., Li, W., Lin, L., 2017. Antibacterial activity of liposome containing curry plant essential oil against Bacillus cereus in rice: Cui et al. J. Food Saf. 37, e12302. https://doi.org/10.1111/jfs.12302

Dag, D., Oztop, M.H., 2017. Formation and Characterization of Green Tea Extract Loaded Liposomes: Formation and characterization... J. Food Sci. 82, 463-470. https://doi.org/10.1111/1750-3841.13615

Detoni, C.B., Cabral-Albuquerque, E.C.M., Hohlemweger, S.V.A., Sampaio, C., Barros, T.F., Velozo, E.S., 2009. Essential oil from Zanthoxylum tingoassuiba loaded into multilamellar liposomes useful as antimicrobial agents. J. Microencapsul. 26, 684-691. https://doi.org/10.3109/02652040802661887 
Gharib, R., Auezova, L., Charcosset, C., Greige-Gerges, H., 2018. Effect of a series of essential oil molecules on DPPC membrane fluidity: a biophysical study. J. Iran. Chem. Soc. 15, 75-84. https://doi.org/10.1007/s13738-017-1210-1

Gharib, R., Najjar, A., Auezova, L., Charcosset, C., Greige-Gerges, H., 2017. Interaction of Selected Phenylpropenes with Dipalmitoylphosphatidylcholine Membrane and Their Relevance to Antibacterial Activity. J. Membr. Biol. 250, 259-271. https://doi.org/10.1007/s00232-017-9957-y

Gortzi, O., Lala, S., Chinou, I., Tsaknis, J., 2007. Evaluation of the Antimicrobial and Antioxidant Activities of Origanum dictamnus Extracts before and after Encapsulation in Liposomes. Molecules 12, 932-945. https://doi.org/10.3390/12050932

Habib, L., Khreich, N., Jraij, A., Abbas, S., Magdalou, J., Charcosset, C., Greige-Gerges, H., 2013. Preparation and characterization of liposomes incorporating cucurbitacin E, a natural cytotoxic triterpene. Int. J. Pharm. 448, 313-319. https://doi.org/10.1016/j.ijpharm.2013.03.027

Hajhashemi, V., Ghannadi, A., Sharif, B., 2003. Anti-inflammatory and analgesic properties of the leaf extracts and essential oil of Lavandula angustifolia Mill. J. Ethnopharmacol. 89, 6771. https://doi.org/10.1016/S0378-8741(03)00234-4

Hyldgaard, M., Mygind, T., Meyer, R.L., 2012. Essential Oils in Food Preservation: Mode of Action, Synergies, and Interactions with Food Matrix Components. Front. Microbiol. 3. https://doi.org/10.3389/fmicb.2012.00012

Hyldgaard, M., Mygind, T., Piotrowska, R., Foss, M., Meyer, R.L., 2015. Isoeugenol has a nondisruptive detergent-like mechanism of action. Front. Microbiol. 6. https://doi.org/10.3389/fmicb.2015.00754

Ibarguren, M., Alonso, A., Tenchov, B.G., Goñi, F.M., 2010. Quantitation of cholesterol incorporation into extruded lipid bilayers. Biochim. Biophys. Acta BBA - Biomembr. 1798, 1735-1738. https://doi.org/10.1016/j.bbamem.2010.06.004

Isman, M.B., Wilson, J.A., Bradbury, R., 2008. Insecticidal Activities of Commercial Rosemary Oils ( Rosmarinus officinalis .) Against Larvae of Pseudaletia unipuncta . and Trichoplusia $n i$. in Relation to Their Chemical Compositions. Pharm. Biol. 46, 82-87. https://doi.org/10.1080/13880200701734661

Jaafar-Maalej, C., Diab, R., Andrieu, V., Elaissari, A., Fessi, H., 2010. Ethanol injection method for hydrophilic and lipophilic drug-loaded liposome preparation. J. Liposome Res. 20, 228-243. https://doi.org/10.3109/08982100903347923

Jaganathan, S.K., Mazumdar, A., Mondhe, D., Mandal, M., 2011. Apoptotic effect of eugenol in human colon cancer cell lines. Cell Biol. Int. 35, 607-615. https://doi.org/10.1042/CBI20100118

Kaddah, S., Khreich, N., Kaddah, F., Charcosset, C., Greige-Gerges, H., 2018. Cholesterol modulates the liposome membrane fluidity and permeability for a hydrophilic molecule. Food Chem. Toxicol. 113, 40-48. https://doi.org/10.1016/j.fct.2018.01.017

Kaefer, C.M., Milner, J.A., 2008. The role of herbs and spices in cancer prevention. J. Nutr. Biochem. 19, 347-361. https://doi.org/10.1016/j.jnutbio.2007.11.003

Khosravi-Darani, K., Khoosfi, M.E., Hosseini, H., 2016. Encapsulation of Zataria multiflora Boiss. Essential Oil in Liposome: Antibacterial Activity Against E. Coli O157:H7 in Broth Media and Minced Beef: ANTIBACTERIAL ACTIVITY OF LIPOSOMAL ESSENTIAL OIL. J. Food Saf. 36, 515-523. https://doi.org/10.1111/jfs.12271 
Lin, L., Zhang, X., Zhao, C., Cui, H., 2016. Liposome containing nutmeg oil as the targeted preservative against Listeria monocytogenes in dumplings. RSC Adv. 6, 978-986. https://doi.org/10.1039/C5RA22098J

Liolios, C.C., Gortzi, O., Lalas, S., Tsaknis, J., Chinou, I., 2009. Liposomal incorporation of carvacrol and thymol isolated from the essential oil of Origanum dictamnus L. and in vitro antimicrobial activity. Food Chem. 112, 77-83. https://doi.org/10.1016/j.foodchem.2008.05.060

Majeed, H., Bian, Y.-Y., Ali, B., Jamil, A., Majeed, U., Khan, Q.F., Iqbal, K.J., Shoemaker, C.F., Fang, Z., 2015. Essential oil encapsulations: uses, procedures, and trends. RSC Adv. 5, 58449-58463. https://doi.org/10.1039/C5RA06556A

Miguel, M.G., 2010. Antioxidant and Anti-Inflammatory Activities of Essential Oils: A Short Review. Molecules 15, 9252-9287. https://doi.org/10.3390/molecules 15129252

Moghimipour, E., Aghel, N., Zarei Mahmoudabadi, A., Ramezani, Z., Handali, S., 2012. Preparation and Characterization of Liposomes Containing Essential Oil of Eucalyptus camaldulensis Leaf. Jundishapur J. Nat. Pharm. Prod. 7, 117-122.

Nasr, G., Greige-Gerges, H., Elaissari, A., Khreich, N., 2020. Liposomal membrane permeability assessment by fluorescence techniques: main permeabilizing agents, applications and challenges. Int. J. Pharm. 119198. https://doi.org/10.1016/j.ijpharm.2020.119198

Nazzaro, F., Fratianni, F., De Martino, L., Coppola, R., De Feo, V., 2013. Effect of Essential Oils on Pathogenic Bacteria. Pharmaceuticals 6, 1451-1474. https://doi.org/10.3390/ph6121451

Raut, J.S., Karuppayil, S.M., 2014. A status review on the medicinal properties of essential oils. Ind. Crops Prod. 62, 250-264. https://doi.org/10.1016/j.indcrop.2014.05.055

Schnitzler, P., Neuner, A., Nolkemper, S., Zundel, C., Nowack, H., Sensch, K.H., Reichling, J., 2010. Antiviral Activity and Mode of Action of Propolis Extracts and Selected Compounds. Phytother. Res. 24, S20-S28. https://doi.org/10.1002/ptr.2868

Sebaaly, C., Greige-Gerges, H., Agusti, G., Fessi, H., Charcosset, C., 2015a. Large-scale preparation of clove essential oil and eugenol-loaded liposomes using a membrane contactor and a pilot plant. J. Liposome Res. 1-13. https://doi.org/10.3109/08982104.2015.1057849

Sebaaly, C., Jraij, A., Fessi, H., Charcosset, C., Greige-Gerges, H., 2015b. Preparation and characterization of clove essential oil-loaded liposomes. Food Chem. 178, 52-62. https://doi.org/10.1016/j.foodchem.2015.01.067

Sen, R., Sahoo, S., Satpathy, S., 2014. Liposomes as drug delivery system: A brief review. Int. J. Res. Pharm. Sci. 5, 309-321.

Sherry, M., Charcosset, C., Fessi, H., Greige-Gerges, H., 2013. Essential oils encapsulated in liposomes: a review. J. Liposome Res. 23, 268-275. https://doi.org/10.3109/08982104.2013.819888

Teixeira, B., Marques, A., Ramos, C., Serrano, C., Matos, O., Neng, N.R., Nogueira, J.M.F., Saraiva, J.A., Nunes, M.L., 2013. Chemical composition and bioactivity of different oregano ( Origanum vulgare ) extracts and essential oil: Composition and bioactivity of oregano products. J. Sci. Food Agric. 93, 2707-2714. https://doi.org/10.1002/jsfa.6089

Tian, J., Ban, X., Zeng, H., He, J., Chen, Y., Wang, Y., 2012. The Mechanism of Antifungal Action of Essential Oil from Dill (Anethum graveolens L.) on Aspergillus flavus. PLoS ONE 7, e30147. https://doi.org/10.1371/journal.pone.0030147 
Trombetta, D., Castelli, F., Sarpietro, M.G., Venuti, V., Cristani, M., Daniele, C., Saija, A., Mazzanti, G., Bisignano, G., 2005. Mechanisms of Antibacterial Action of Three Monoterpenes. Antimicrob. Agents Chemother. 49, 2474-2478. https://doi.org/10.1128/AAC.49.6.2474-2478.2005

Varona, S., Martín, Á., Cocero, M.J., 2011. Liposomal Incorporation of Lavandin Essential Oil by a Thin-Film Hydration Method and by Particles from Gas-Saturated Solutions. Ind. Eng. Chem. Res. 50, 2088-2097. https://doi.org/10.1021/ie102016r

Yoshida, P.A., Yokota, D., Foglio, M.A., Rodrigues, R.A.F., Pinho, S.C., 2010. Liposomes incorporating essential oil of Brazilian cherry (Eugenia uniflora L.): Characterization of aqueous dispersions and lyophilized formulations. J. Microencapsul. 27, 416-425. https://doi.org/10.3109/02652040903367327

Zhang, L.-L., Zhang, L.-F., Xu, J.-G., Hu, Q.-P., 2017. Comparison study on antioxidant, DNA damage protective and antibacterial activities of eugenol and isoeugenol against several foodborne pathogens. Food Nutr. Res. 61, 1353356. https://doi.org/10.1080/16546628.2017.1353356

Zhang, Y., Liu, X., Wang, Y., Jiang, P., Quek, S., 2016. Antibacterial activity and mechanism of cinnamon essential oil against Escherichia coli and Staphylococcus aureus. Food Control 59, 282-289. https://doi.org/10.1016/j.foodcont.2015.05.032 